\title{
Trailed vorticity modeling for aeroelastic wind turbine simulations in standstill
}

\author{
Georg R. Pirrung ${ }^{1}$, Helge A. Madsen ${ }^{1}$, and Scott Schreck ${ }^{2}$ \\ ${ }^{1}$ Wind Energy Department, Technical University of Denmark, Frederiksborgvej 399, 4000 Roskilde, Denmark \\ ${ }^{2}$ National Renewable Energy Laboratory, 15013 Denver West Parkway, Golden, CO 80401, USA \\ Correspondence to: Georg R. Pirrung (gepir@dtu.dk)
}

Received: 7 January 2017 - Discussion started: 6 March 2017

Revised: 6 September 2017 - Accepted: 10 October 2017 - Published: 20 November 2017

\begin{abstract}
Current fast aeroelastic wind turbine codes suitable for certification lack an induction model for standstill conditions. A trailed vorticity model previously used as an addition to a blade element momentum theory based aerodynamic model in normal operation has been extended to allow computing the induced velocities in standstill. The model is validated against analytical results for an elliptical wing in constant inflow and against standstill measurements from the NREL/NASA Phase VI unsteady experiment. The extended model obtains good results in the case of the elliptical wing but underpredicts the steady loading for the Phase VI blade in attached flow. The prediction of the dynamic force coefficient loops from the Phase VI experiment is improved by the trailed vorticity modeling in both attached flow and stall in most cases. The exception is the tangential force coefficient in stall, where the codes and measurements deviate and no clear improvement is visible. This article also contains aeroelastic simulations of the DTU $10 \mathrm{MW}$ reference turbine in standstill at turbulent inflow with a fixed and idling rotor. The influence of the trailed vorticity modeling on the extreme flapwise blade root bending moment is found to be small.
\end{abstract}

\section{Introduction}

State-of-the-art aeroelastic wind turbine codes that are suitable for simulating the many time series needed for certification typically use an aerodynamics model based on blade element momentum (BEM) theory. These BEM-based models can be extended by tip loss corrections and so-called dynamic inflow models that take the wake inertia into account. With this extension, they are suitable for predicting the varying induced velocities in an unsteady aeroelastic simulation. In addition to the dynamic induced velocities, there are also dynamic effects due to shed vorticity and dynamic stall, which occur on faster timescales than the dynamic inflow and are typically taken into account by 2-D unsteady airfoil aerodynamics models, in this work the one described in Hansen et al. (2004).

Thus, both the larger-scale wake effects and the smallerscale unsteady airfoil aerodynamics are taken into account if the turbine is in operation. In standstill, however, BEM theory cannot be used because the basic assumption in BEM, i.e., that the rotor can be approximated by a disc, is violated. Therefore, the induced velocities due to the vortices trailed from the blades are not modeled, which results in both a wrong steady-state load distribution and missing dynamics.

Wind turbine blades are twisted to ensure a reasonable angle of attack distribution along the blade in operation. In standstill, on the other hand, the blade twist leads to large load variations along the blade and thus strong trailed vorticity that is not modeled in the aeroelastic codes used for wind turbine certification. Further, the inflow turbulence, which in normal operation only affects a part of the relative flow velocity at the airfoils (the other part being due to rotor rotation), causes very large dynamic variations in the angle of attack (AOA) along the blade in standstill. In idling conditions a yaw error, as well as nacelle tilt and wind inclination, is directly translated into AOA variations as the blades rotate slowly.

In this work, a trailed vorticity model, which was originally designed for normal operation and implemented as 
part of a BEM-based model in the aeroelastic code HAWC2, (Larsen and Hansen, 2015; Pirrung et al., 2016, 2017), has been extended so that it can be used in standstill conditions. Results from this extended model are compared to the analytical constant downwash at an elliptical wing and measurements from the NREL/NASA Phase VI unsteady experiment, (Hand et al., 2001). Aeroelastic computations in standstill with turbulent inflow are performed to evaluate the influence of the model on radial load distributions and extreme flapwise blade root bending moment.

\section{Near-wake model description}

The near-wake model (NWM) for trailed vorticity was originally developed for use in helicopter aerodynamics. It was assumed in the original model that the trailed vorticity stays in the rotor plane. The induced velocity at a blade section due to a trailed vortex element decreases as that vortex element moves away from the blade. This decreasing induction is approximated by exponential functions. This approximation makes it possible to use an indicial function algorithm to avoid the time-consuming numerical integration of vortex arcs based on the Biot-Savart law. The model has since been modified to enable the computation of the induction due to trailed helical vortex arcs (Pirrung et al., 2016), which is important in normal operation at high wind speed. Further, it has been shown by Pirrung et al. (2017) that using one exponential function instead of two is possible with negligible accuracy loss.

A sketch of the near-wake geometry is shown in Fig. 1. The induction $W$ at a blade section $s$ at a time step $i$ is found as the sum of the induced velocities due to all vortex arcs $v$ trailed from a blade:

$W_{s}^{i}=\sum_{v=1}^{N_{v}} W_{s, v}^{i}$.

The induced velocity due to an individual vortex arc is

$W_{s, v}^{i}=W_{s, v}^{i-1} e^{-\Delta \beta / \Phi_{s, v}}+D_{w, s, v} \Delta \Gamma\left(1-e^{-\Delta \beta / \Phi_{s, v}}\right)$,

where $\Delta \beta$ is the angle the blade rotates during a time step and $\Phi$ is a geometric parameter depending on the positions of vortex trailing point and blade section, as well as the helix angle of the trailed vortex arc. The trailed vortex strength, which depends on the radial gradient of the bound circulation, is $\Delta \Gamma$, and $D_{w, s, v}$ describes the induced velocity at section $s$ due to a trailed vortex arc $v$ with circulation 1 that starts directly at the blade. The advantage of using exponential functions is apparent: to obtain the induction at a new time step, the induction due to the newly trailed vortex element, the right term in Eq. (2), is added to the exponentially decreasing induced velocity due to all previously trailed elements contained in $W_{s, v}^{i-1}$.

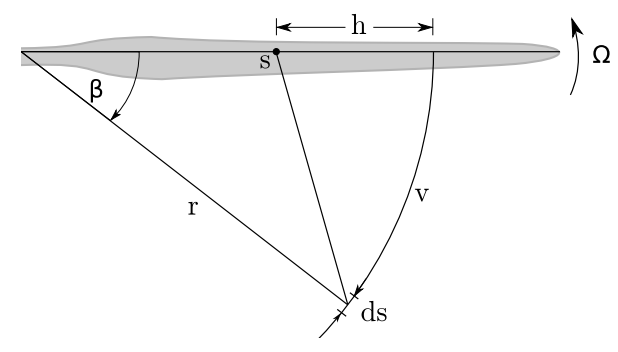

Figure 1. Sketch of the geometry in the near wake. The vortex arc $v$, trailed at radius $r$, induces axial (out-of-plane) and tangential (inplane) velocities at the section $s$. The radial distance between vortex trailing point and section position is denoted $h$ and $h>0$ if the vortex is trailed outboard of the section. The angle $\beta$ describes how far a vortex element with length $\mathrm{d} s$ has moved away from the blade. The figure is adapted from Pirrung et al. (2014). In practice, the blade is discretized into many sections and vortices are trailed from the root and tip and in between sections.

\section{Model extension}

In order to enable the computation of standstill cases, a new definition of the angle $\beta$ is necessary. The previous implementation used the projection of the trailed vortex filament in the rotor plane, which is not possible in standstill conditions. Thus, the angle $\beta$ is redefined:

$\beta^{*}=\frac{v_{\mathrm{rel}} \Delta t}{r}$,

where $v_{\text {rel }}$ is the relative flow velocity at the radial station from which the vortex filament is trailed. If the trailed vorticity stays in the rotor plane, the old and new definitions are identical: $\beta=\beta^{*}$.

The new definition of $\beta^{*}$ accounts for the differing element length trailed in one time step due to the downwind convection velocity. The axial and tangential components of the induced velocities due to the newest element (cf. Eq. 2), can be determined based on the helix angle $\varphi$ :

$D_{w, s, v, \text { axial }}=D_{w, s, v} \cos \varphi$,

$D_{w, s, v, \text { tangential }}=D_{w, s, v} \sin \varphi$.

Because the near-wake model is mainly meant to capture trailed vorticity effects close to the blade, the local inflow angle is used as helix angle $\varphi$. This inflow angle is computed based on the velocity triangle at the vortex trailing point and is affected by the free wind speed including turbulence, the movement of the blade and the induced velocities due to near and far wake. This way the near-wake flow situation depends only on the velocities at the blade section, which is similar to how the 2-D unsteady aerodynamics effects are computed; see Hansen et al. (2004). The time simulation of axial and tangential induction is then computed independently, so Eqs. (1) and (2) are evaluated twice for each section-vortexarc combination. 
If the downwind convection velocity increases, the paths of the trailed vorticity change from circular (at zero convection speed) over helical (at moderate convection speed) to straight (at standstill). This influences both the steady-state value of the induction from trailed vorticity and the dynamic behavior. Both of these can be modeled by changing the parameter $\Phi$ in Eq. (2).

As described in Pirrung et al. (2016), an optimal value of $\Phi$ can be computed. With this $\Phi_{\text {opt }}$, the indicial function approximation computes the same steady-state induced velocity as the Biot-Savart law:

$$
\begin{aligned}
& \int_{0}^{\pi / 2}\left(\frac{\left(\frac{h}{r}\right)^{2}\left[1-\left(1-\frac{h}{r}\right) \cos \beta\right]}{\left(1+\left(1-\frac{h}{r}\right)^{2}-2\left(1-\frac{h}{r}\right) \cos \beta+(\beta \tan \varphi)^{2}\right)^{3 / 2}}\right) \mathrm{d} \beta \\
& =\int_{0}^{\pi / 2}\left(1.359 e^{-\beta / \Phi_{\mathrm{opt}}}-0.359 e^{-4 \beta / \Phi_{\mathrm{opt}}}\right) \mathrm{d} \beta
\end{aligned}
$$

Here, $r$ denotes the radial position where the vortex is trailed and $h$ the distance from the vortex trailing position to the radial position of the blade section where the induction is to be determined (positive if the section is inboard the vortex); cf. Fig. 1. For straight vortices, $\Phi$ varies linearly with $h / r$ :

$\Phi_{\mathrm{s}}=\left\{\begin{array}{cl}0.788 \frac{h}{r} & \text { for } 0<h / r<1 \\ -0.788 \frac{h}{r} & \text { for } h / r<0 .\end{array}\right.$

To ensure that the model can be used for straight vortices in standstill conditions and helical vortices in normal operation, a new $\Phi^{*}$ is computed, that is a linear interpolation between $\Phi_{\mathrm{S}}$ for straight vortices Eq. (7) and the expression of Wang and Coton for circular vortices, Wang and Coton (1999):

$\Phi^{*}=k_{\Phi} \Phi_{\mathrm{S}}+\left(1-k_{\Phi}\right) \Phi$,

where the interpolation $k_{\Phi}$ is a function of both $h / r$ and the tangent of the helix angle. The straight and circular $\Phi$ approach each other for $h / r \rightarrow 0$, meaning for sections very close to vortex trailing points, where the influence of the vortex is large and an accurate computation of $\Phi$ is thus very important. Therefore, the interpolation proposed in Eq. (8) ensures good results for close positions, which would be difficult to achieve by direct curve fitting of $\Phi$ to the optimal value according to Eq. (6).

For positive values of $h / r, k_{\Phi}$ can be approximated as

$k_{\Phi}=a_{h r, 1}+a_{h r, 2} \varphi+a_{h r, 3} \varphi^{2}+a_{h r, 4} \varphi^{3}$,

$a_{h r, i}=p_{i, 1}+p_{i, 2} \frac{h}{r}+p_{i, 3}\left(\frac{h}{r}\right)^{2}+p_{i, 4}\left(\frac{h}{r}\right)^{3}$.

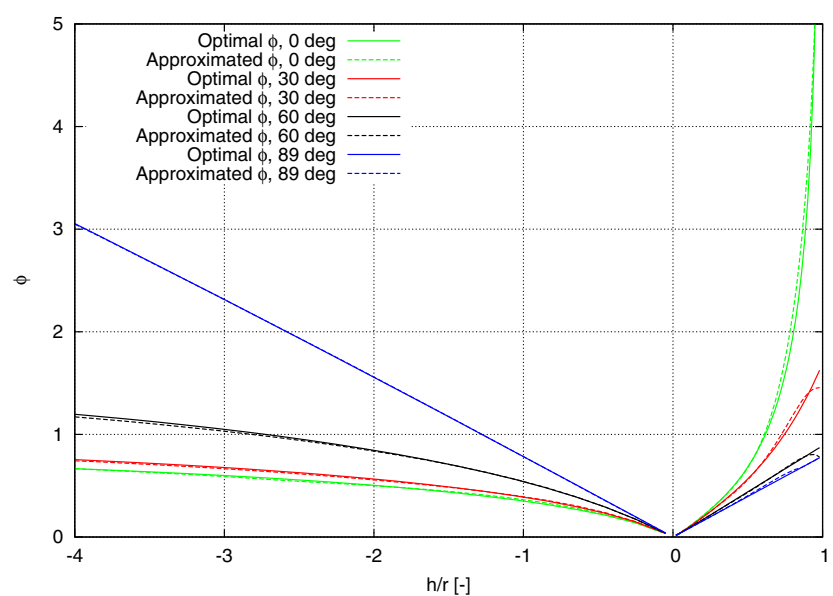

Figure 2. Approximation of $\Phi$ at different helix angles compared to the optimal $\Phi$ value.

For negative values of $h / r$

$$
\begin{aligned}
& k_{\Phi}=a_{h r, 1}+a_{h r, 2} e^{a_{h r, 3}\left(\frac{\pi}{2}-\varphi\right)}+a_{h r, 4} e^{-8\left(\frac{\pi}{2}-\varphi\right)} \\
& \quad-a_{h r, 2}-a_{h r, 4}, \\
& a_{h r, i}=n_{i, 1}+n_{i, 2} e^{n_{i, 3}\left(\frac{h}{r}\right)}+n_{i, 4} e^{n_{i, 5}\left(\frac{h}{r}\right)}-n_{i, 4}-n_{i, 2} .
\end{aligned}
$$

The values $n_{i, j}$ and $p_{i, j}$ are collected in the matrices $\mathbf{N}$ and P:

$$
\begin{aligned}
\mathbf{N} & =\left(\begin{array}{ccccc}
1.01933 & -0.13567 & 0.39552 & 0.08018 & 44.83475 \\
12.98745 & 50.0 & 0.00235 & 11.31161 & 3935.34323 \\
-0.69016 & 101.23878 & -0.00154 & 3.99520 & 0.39454 \\
-0.26925 & 50.0 & -0.00248 & 0.40364 & 1.16610
\end{array}\right), \\
\mathbf{P} & =\left(\begin{array}{cccc}
-1.64637 & 8.14821 & -12.17849 & 5.02653 \\
-0.49901 & 6.08465 & -15.17120 & 14.82541 \\
3.90836 & -18.76623 & 39.12433 & -29.48701 \\
-1.60623 & 7.42953 & -15.85948 & 11.68702
\end{array}\right) .
\end{aligned}
$$

Optimal and approximated values for $\Phi$ are shown for different helix angles ranging from 0 (circular arcs) to $89^{\circ}$ in Fig. 2. The $89^{\circ}$ have been chosen because for $90^{\circ}$ the integral in Eq. (6) cannot be evaluated. It is shown clearly that the approximation gives a good representation of $\Phi$ across the range of helix angles. There are some deviations for $h / r \rightarrow 1$, which represents the influence of vortices close to the tip on the root sections. Because the deviations only influence roughly the innermost $5 \%$ of the rotor radius, where often no aerodynamic profiles are installed, the quality of the approximation is acceptable.

\section{Unsteady airfoil aerodynamics model}

The 2-D unsteady airfoil aerodynamics model in HAWC2 consists of both an attached flow model for the 2-D shed vorticity effects and a dynamic stall model to predict unsteady flow separation, as described in Hansen et al. (2004). The attached flow model uses indicial functions assuming a flat plate. The dynamic stall model interpolates between a 
fully attached and fully separated airfoil polar, based on a time-lagged trailing edge separation point. The dynamic stall model does not include leading-edge separation.

\section{Interaction between dynamic stall model and trailed vorticity model}

The trailed vortex strength $\Delta \Gamma$ in the near-wake model (see Eq. 2) is given by the difference in bound circulation between the adjacent sections to a vortex trailing point. The near-wake model needs to be iterated to convergence. The bound circulation is part of that iteration loop, including attached flow airfoil aerodynamics effects. Inside that loop, the quasi steady bound circulation is computed according to the quasi steady lift coefficient (Pirrung et al., 2017). This accounts for stall in the bound circulation computation. The converged induced velocity due to the trailed vorticity is then used to compute the angle of attack, which is the input to the unsteady airfoil aerodynamics model. The unsteady airfoil aerodynamics model then computes the effective angle of attack and the influence of dynamic stall on the aerodynamic forces. The only deviation from the basic structure of the implementation from the structure outlined in Fig. 3 of the article by Pirrung et al. (2017) is that no far-wake model is used because the BEM modeling is not valid in standstill conditions and the near-wake model computes the full induction due to the semi-infinite trailed vorticity behind the blades.

\section{Results}

\subsection{Elliptical wing}

The case of an elliptical wing with a $10 \mathrm{~m}$ span has been used previously to test the NWM (Madsen and Rasmussen, 2004; Pirrung et al., 2014). In these earlier publications, the wing was placed at the end of a very long, slowly rotating blade to ensure an almost parallel inflow. In this work, the wing is instead mounted on a $0.5 \mathrm{~m}$ long, nonrotating hub in a uniform inflow of $35 \mathrm{~m} \mathrm{~s}^{-1}$. The previous publications prescribed an elliptical circulation distribution, but in this work the wing is modeled with a geometric AOA to the inflow of $5.45^{\circ}$ and a maximum chord length of $5.21 \mathrm{~m}$. The geometric AOA is defined as the angle of the local chord line with respect to the inflow direction in HAWC2, which corresponds to the wind tunnel center line in the case of the Phase VI measurements discussed later. A lift gradient of $2 \pi$ is used, which leads to the analytical result of a constant downwash of $1.5 \mathrm{~m} \mathrm{~s}^{-1}$ at the wing.

Figure 3 compares downwash at the lifting line computed from original and extended NWM with the analytical solution. The original model fails to predict the constant downwash in standstill, while the results from the extended model are in good agreement with the analytical solution.

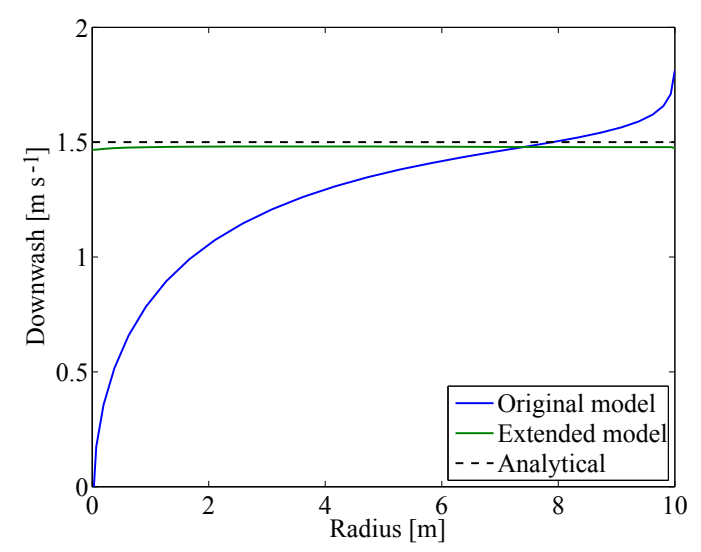

Figure 3. Results for an elliptical wing.

\subsection{NREL Phase VI rotor in standstill}

In all following comparisons, "HAWC2" refers to HAWC2 standstill simulations. The BEM model and dynamic inflow model are disabled because the BEM model is not valid in standstill and the dynamic inflow model simulates the unsteady behavior of the BEM induction. The 2-D unsteady aerodynamics model containing shed vorticity and dynamic stall modeling as introduced in Sect. 4 is active.

In addition to the 2-D unsteady aerodynamics model, the "HAWC2 NW" simulations include the trailed vorticity modeling by the extended near-wake model.

\subsubsection{Constant pitch angle}

Besides measurements at operation, the Phase VI experiment also contained measurements in standstill, some of which have been compared to computational fluid dynamics (CFD) results by Johansen et al. (2002) and Sørensen and Schreck (2012). Here, some steady comparisons with measurements published in Johansen et al. (2002) are shown together with comparisons at a lower geometric angle of attack.

The inflow speed in the cases presented here is $20 \mathrm{~m} \mathrm{~s}^{-1}$, which results in a Reynolds number of 0.86 million at $47 \%$ blade radius. The Reynolds number varies along the blade with the chord length (ignoring induced velocity effects on the Reynolds number), and the aerodynamic code interpolates accordingly between different airfoil polars.

A comparison of the radial distribution of the normal force coefficient is shown in Fig. 4 at 3.5 and $18.2^{\circ}$ geometric AOA at $47 \%$ blade radius. At $3.5^{\circ}$ geometric AOA at the $47 \%$ station, most of the blade is in attached flow. In this case, the near-wake model predicts a radial distribution of the normal coefficient that agrees well with the measurements in terms of the radial load gradients, but there is an offset to the measurements. No explanation for this offset has been found. The results at the higher geometric AOA, where most of the blade is in stall, are shown in Fig. 4b. In this case the near-wake model can predict the root vortex well, but the agreement 

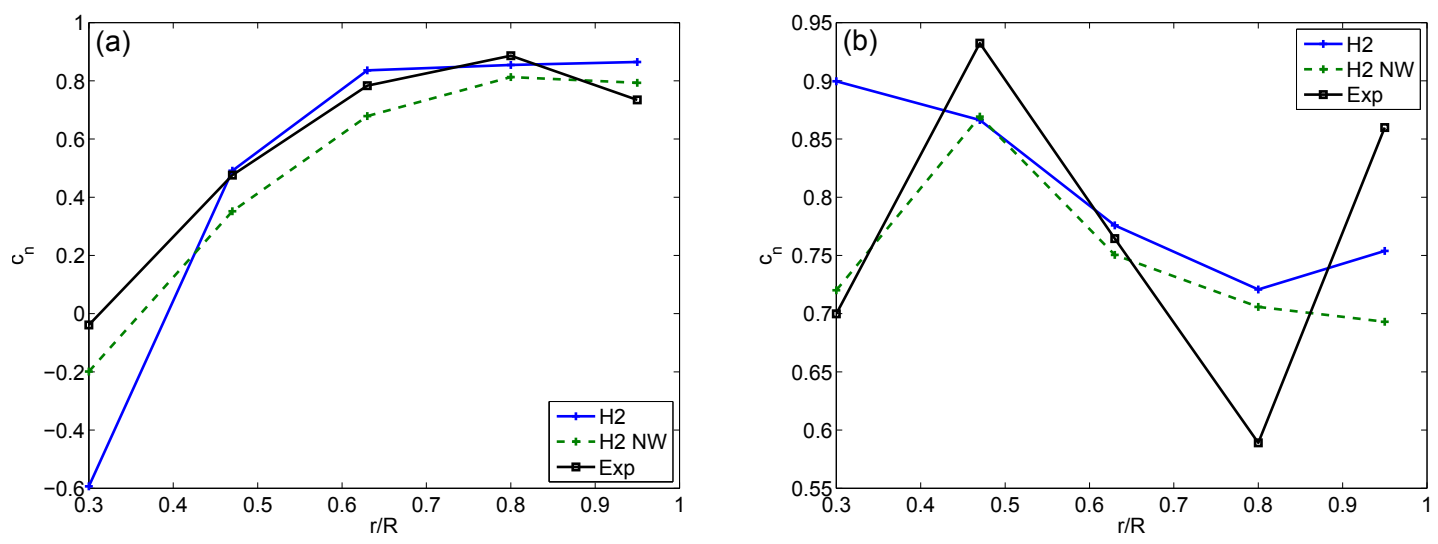

Figure 4. Radial distribution of normal force coefficients at 3.5 (a) and 18.2 (b) degrees geometric AOA at $47 \%$ blade radius. Results from HAWC2 (denoted H2) and HAWC2 including the extended near-wake model presented in this paper (H2 NW) are compared to measurements.

with the measurements becomes worse toward the tip, where the blade is in deep stall.

The steady-state comparison of the tangential force coefficients in these cases in Fig. 5 leads to the same conclusions. Again there appears to be an offset between near-wake computations and measurements in the $3.5^{\circ}$ case. In the $18.2^{\circ}$ case the prediction of the root vortex by the near-wake model is clear, but the agreement gets worse towards the stalled tip.

\subsubsection{Varying pitch angle}

Two cases of a pitching blade are presented here: case O47010 with a mean geometric AOA at the $47 \%$ station of $3^{\circ}$ and a pitching amplitude of $2^{\circ}$ at a frequency of $0.739 \mathrm{~Hz}$ (reduced frequency $K=0.0625$ at $47 \%$ ) and case 047320 with a mean geometric AOA at the $47 \%$ station of $14^{\circ}$ and a pitching amplitude of $5.5^{\circ}$ at a frequency of $1.183 \mathrm{~Hz}$ (reduced frequency $K=0.1$ at $47 \%$ ). The free-stream velocity in both cases is $23.3 \mathrm{~m} \mathrm{~s}^{-1}$.

The normal force coefficient variation for the $\mathrm{O} 47010$ case is shown in Fig. 6. The mean geometric AOA is only half a degree different than in the steady case in Fig. 4, and almost the full blade is in attached flow. The unsteady simulation agrees with the steady simulation in an offset, where the HAWC2 NW results are below the measurements at every station but the blade tip. A comparison of the mean values would thus not lead to new conclusions. To make the comparison of the dynamic behavior easier, the mean values of $c_{n}$ have been subtracted in Fig. 6 . The near-wake modeling leads to improved agreement with the measurements everywhere except at the $63 \%$ station, where the differences between the predicted and measured loops are small. At the other radial stations, HAWC2 NW predicts the loop openings and gradients much better than HAWC2. At the $80 \%$ station, for example, HAWC2 NW predicts the slight loop opening due to beginning separation that is seen in the measurements. The HAWC2 computations show a $c_{n}$ gradient and loop opening that is characteristic of too large a mean AOA. This observation is in conflict with the comparison of the steady-state $c_{n}$ in Fig. 4 . There, the larger values predicted by HAWC2 results are in better agreement with the experimental data at the $80 \%$ section than the HAWC2 NW results.

The $c_{t}$ variations in Fig. 7 show improved simulation results due to the NWM at most blade stations except $47 \%$. Similar as in the $c_{n}$ comparison above, HAWC2 NW predicts gradients and loop openings that are very close to the measurements. The normal force coefficient loops for the O47320 case $\left(14^{\circ}\right.$ mean AOA at the $47 \%$ radial station) are shown in Fig. 8. Because the amplitudes are larger and the mean AOA is higher in this case, the loops are more open and more nonlinear. Therefore, the unsteady aerodynamics model has a larger influence on the mean values of $c_{n}$ and $c_{t}$ than in case $\mathrm{O} 47010$, and it has been chosen not to subtract the mean values in the 047320 results. The flow is only attached at the $30 \%$ radial station (cf. Fig. 8), and there HAWC2 NW predicts the $c_{n}$ gradient more accurately than HAWC2. At the $47 \%$ radial station, HAWC2 NW predicts a slightly higher range of $c_{n}$ that is closer to the measurements. Also, the shape of the loop predicted by HAWC $2 \mathrm{NW}$ agrees better with the measurements than that predicted by HAWC2, but both models do not reach as high maximum $c_{n}$ values as the measurements. At the $63 \%$ station HAWC2 NW predicts a slightly more open loop than HAWC2 up to $17^{\circ}$ geometric AOA, which is in better agreement with the measurements. Also in agreement is the increasing normal force coefficient towards higher AOA, which is not predicted by HAWC2. As at the $47 \%$ station, and also further outboard, the models underpredict the maximum measured $c_{n}$ values. However, the local increase in $c_{n}$ at high AOA in the measurements at the $63 \%$ section appears to be due to leadingedge vortex formation. This effect is not included in the dynamic stall modeling; therefore, this overshoot of $c_{n}$ cannot be predicted by HAWC2. To a lesser extend, the same effect 

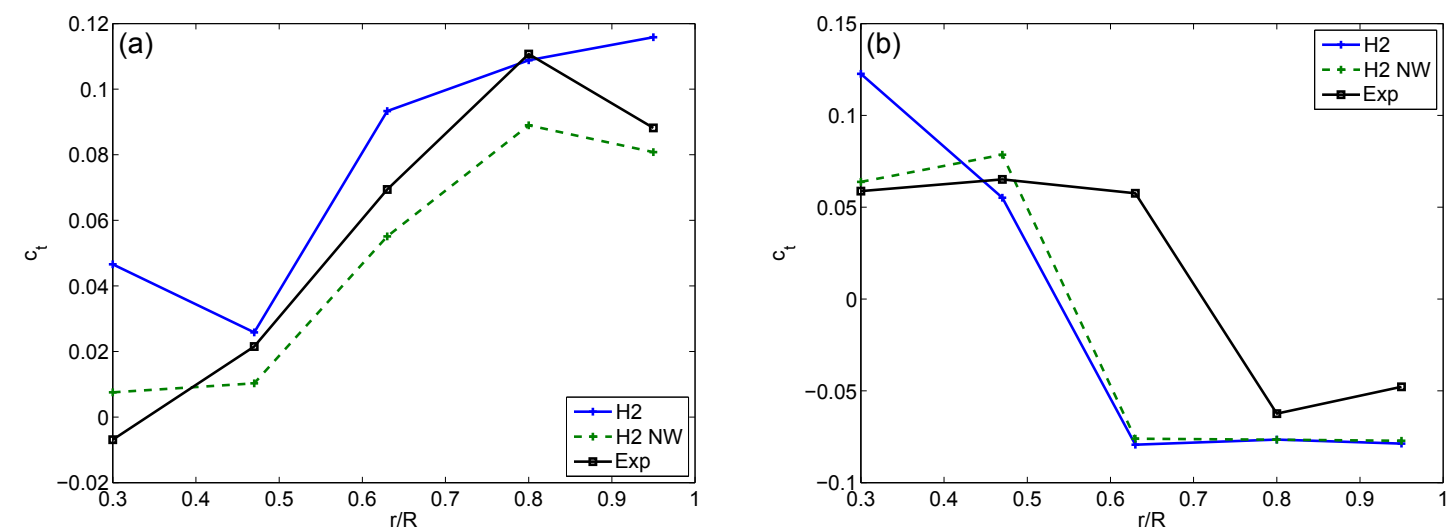

Figure 5. Radial distribution of tangential force coefficients at 3.5 (a) and 18.2 (b) degrees geometric AOA at $47 \%$ blade radius. Results from HAWC2 (denoted H2) and HAWC2 including the extended near-wake model presented in this paper (H2 NW) are compared to measurements.
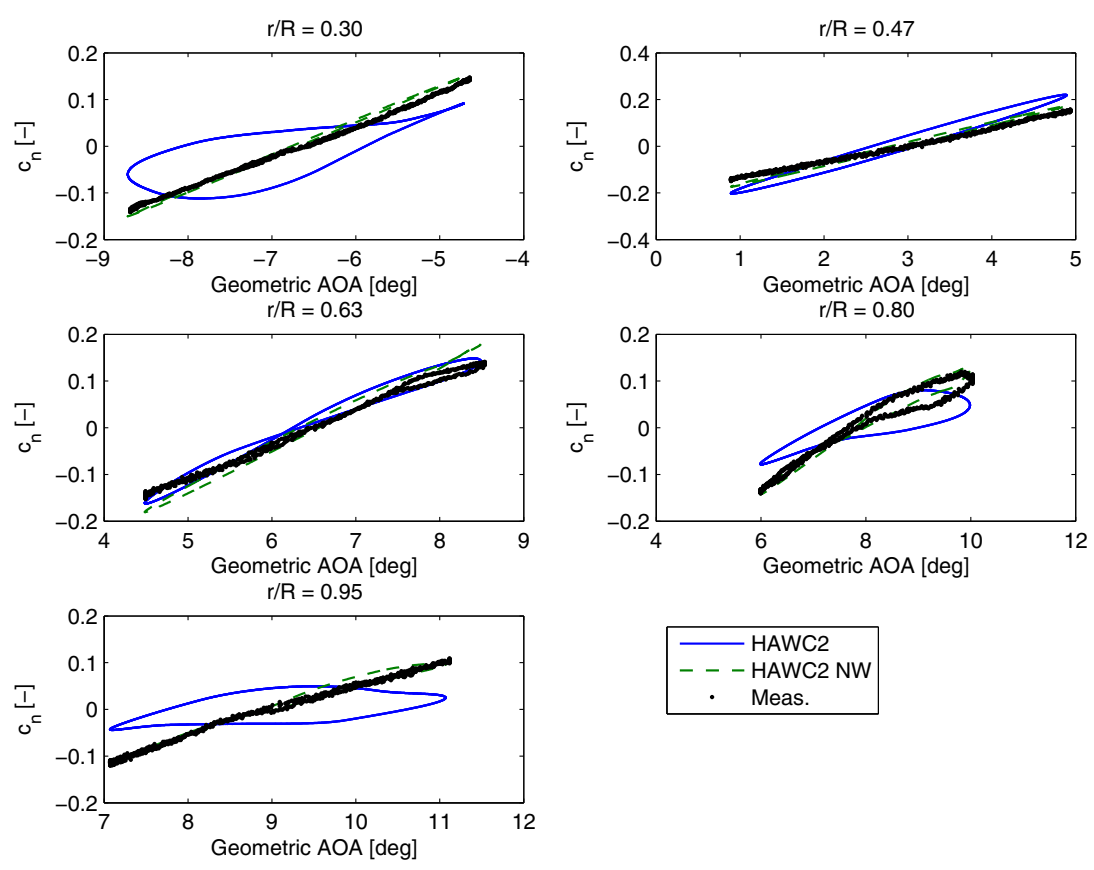

Figure 6. Case O47010. Variation about mean $c_{n}$.

can be seen at the 47 and $80 \%$ stations. At both the 80 and $95 \%$ stations, the loops predicted by HAWC2 are narrowing towards the high angles of attack. This is because the dynamic stall model interpolates between a fully attached and fully separated curve; cf. Sect. 4. At high angles of attack, where the flow is fully separated, the dynamic stall model becomes steady because the separation point does not move any more, and accordingly the loops close. Due to the trailed vorticity in the HAWC2 NW computations, the local angles of attack at the radial stations close to the tip are lower than the geometric angles of attack, and therefore the flow is not yet considered fully separated. Thus, the loops predicted by HAWC2 NW do not become more narrow towards high ge- ometric AOA at the 80 and $95 \%$ stations. However, the narrowing dynamic stall loops are just delayed towards higher geometric AOA.The underlying issue, that the dynamic stall model is not suited for deep stall conditions, remains. Even though the loop opening predicted by HAWC2 NW at the $95 \%$ radial station is closer to the measurements, the gradient of the $c_{n}$ loop cannot be predicted.

The loops of the tangential force coefficient in the $\mathrm{O} 47320$ case are shown in Fig. 9. At the $30 \%$ station in attached flow, HAWC2 NW clearly predicts a loop opening and gradient that agrees better with the measurements than the results from HAWC2. At the $47 \%$ radial station, HAWC2 NW predicts the form of the loop slightly better, but the opening in 

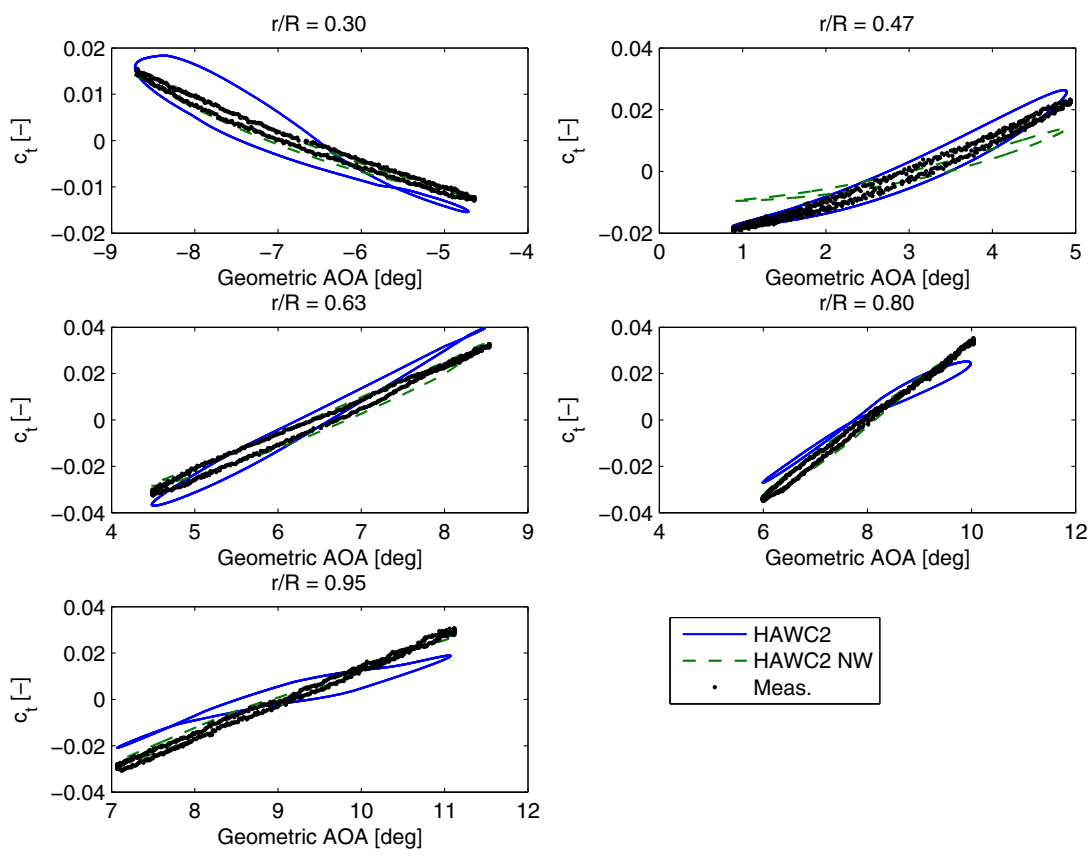

Figure 7. Case O47010. Variation about mean $c_{t}$.

the measured loop is considerably larger. At the further outboard stalled stations, there is generally a large disagreement between both codes and the measurements and it is difficult to state which codes' predictions agree better with the measurements.

\subsection{DTU $10 \mathrm{MW}$ in standstill with turbulent inflow}

Aeroelastic simulations on the DTU $10 \mathrm{MW}$ reference turbine (Bak et al., 2012) have been performed to investigate the effect of the trailed vorticity model in standstill. The mean wind speed in these simulations is $50 \mathrm{~m} \mathrm{~s}^{-1}$, the shear exponent is 0.11 , and the turbulence intensity is $11 \%$. These values are based on design load case (DLC) 6.2 in the design load basis for onshore wind turbines by Hansen et al. (2015). All blades are pitched to $82^{\circ}$, and the simulated time is $700 \mathrm{~s}$, where the first $100 \mathrm{~s}$ are removed to avoid transients. The blades are discretized into 30 equidistantly spaced aerodynamic sections.

The compared codes are HAWC2 with dynamic stall enabled but without induction model and HAWC2 NW, where both dynamic stall and induction due to the trailed vorticity are modeled. In order to enable direct comparisons between the different aerodynamic models a few computations have been performed with a locked rotor and only a single turbulence seed. The radial distributions of AOA, induced velocities, and aerodynamic forces on the blade pointing vertically upward are discussed in Sect. 5.3.1 at wind directions of 0, -15 , and $15^{\circ}$. Wind direction misalignments larger than $15^{\circ}$ are not included in the locked rotor analysis because they can lead to standstill vibrations of the DTU $10 \mathrm{MW}$ (Wang et al.,
2016). These vibrations make it difficult to compare radial load distributions, and their analysis is outside the scope of the present work.

To evaluate the extreme blade root flapwise bending moments, simulations with an idling rotor in the wind direction range of -30 to $30^{\circ}$ ( $5^{\circ}$ resolution) are presented in Sect. 5.3.2. A number of 36 turbulence seeds has been used at each wind direction and for each aerodynamic model. It is important to note that yaw angles outside of the $\pm 15^{\circ}$ range are at the boundaries of the near-wake model validity, which is at its core a simplified lifting line model. The yaw errors up to $\pm 30^{\circ}$ are included in the idling analysis to investigate how the model behaves in these difficult conditions.

\subsubsection{Locked rotor}

The radial distributions of in-plane induced velocity, AOA, and edgewise and flapwise aerodynamic forces are shown in Fig. 10 for a wind direction of $-15^{\circ}$. The solid lines show the mean values, and the standard deviations are indicated by dashed lines. At this wind direction, most of the upward-pointing blade is in negative stall, except for the area around the tip where the flow is attached. Because the nearwake model is implemented such that a bound circulation is computed based on the lift coefficient, a slight reduction in the mean aerodynamic forces can be seen when the nearwake model is enabled. The AOA and induction distributions clearly show the prediction of a root vortex, while the influence of the trailed vorticity on the mean loading toward the blade tip is limited. Because the lift gradient is larger in attached flow, the standard deviation of the induced velocity 

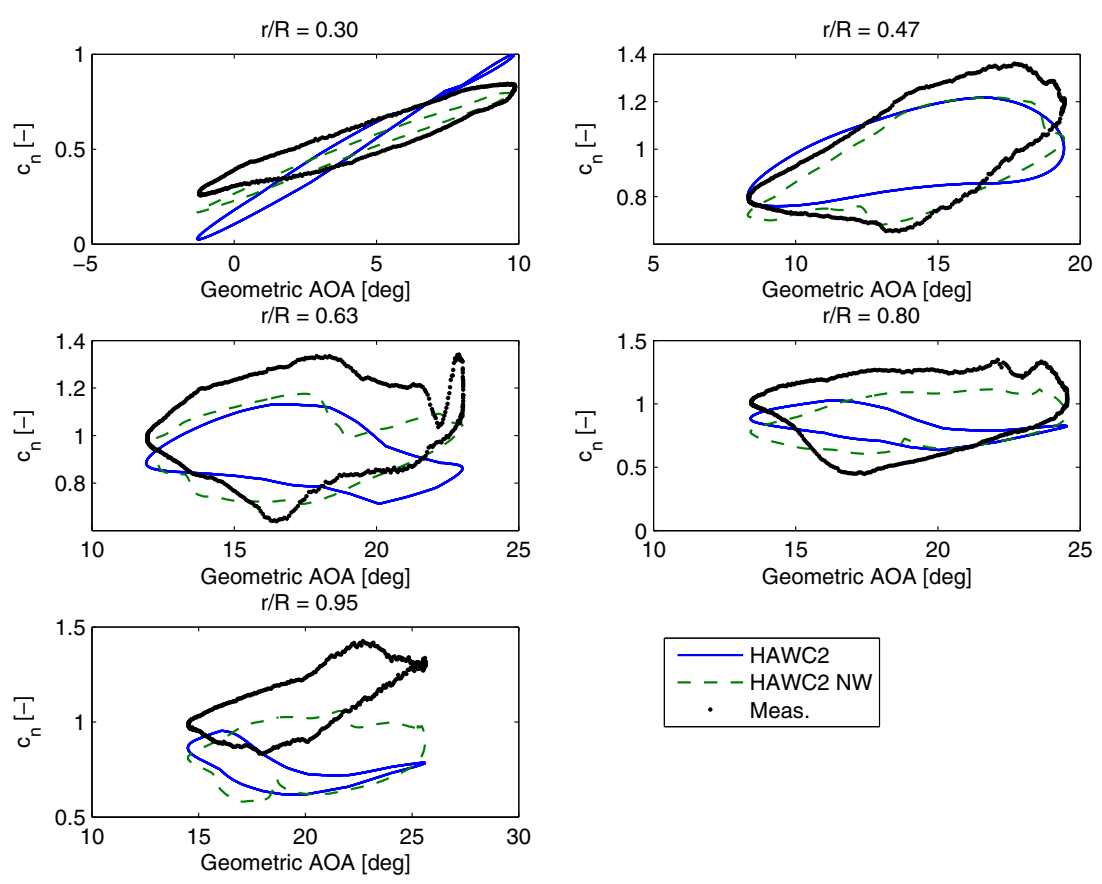

Figure 8. Case $\mathrm{O} 47320 ; c_{n}$.
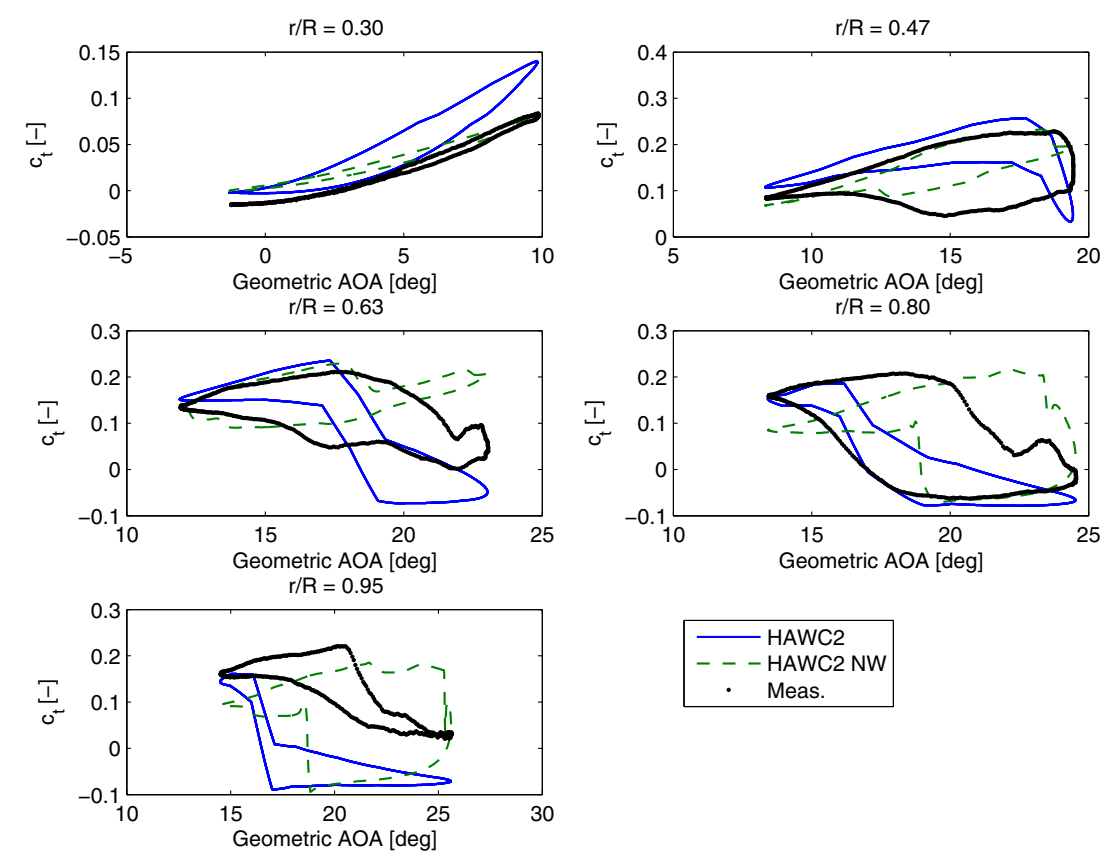

Figure 9. Case O47320; $c_{t}$.

increases towards the tip. These increased induced velocity variations counteract the force variations due to inflow turbulence, which causes slightly smaller standard deviations of the angle of attack and flapwise force if the trailed vorticity modeling is active. There is no clear tip vortex visible in the mean induction distribution because the loading approaches zero towards the tip even without an induction model. Fur- ther the equidistant point spacing means that the small drop in the loading to zero at the very last blade section is not so finely resolved. At $0^{\circ}$ wind direction, the flow is attached on the whole blade; see Fig. 11b. Therefore, the lift gradients are large and the trailed vorticity modeling has a larger influence on the loading. Both the mean aerodynamic forces as well as the standard deviations of these forces are reduced along 

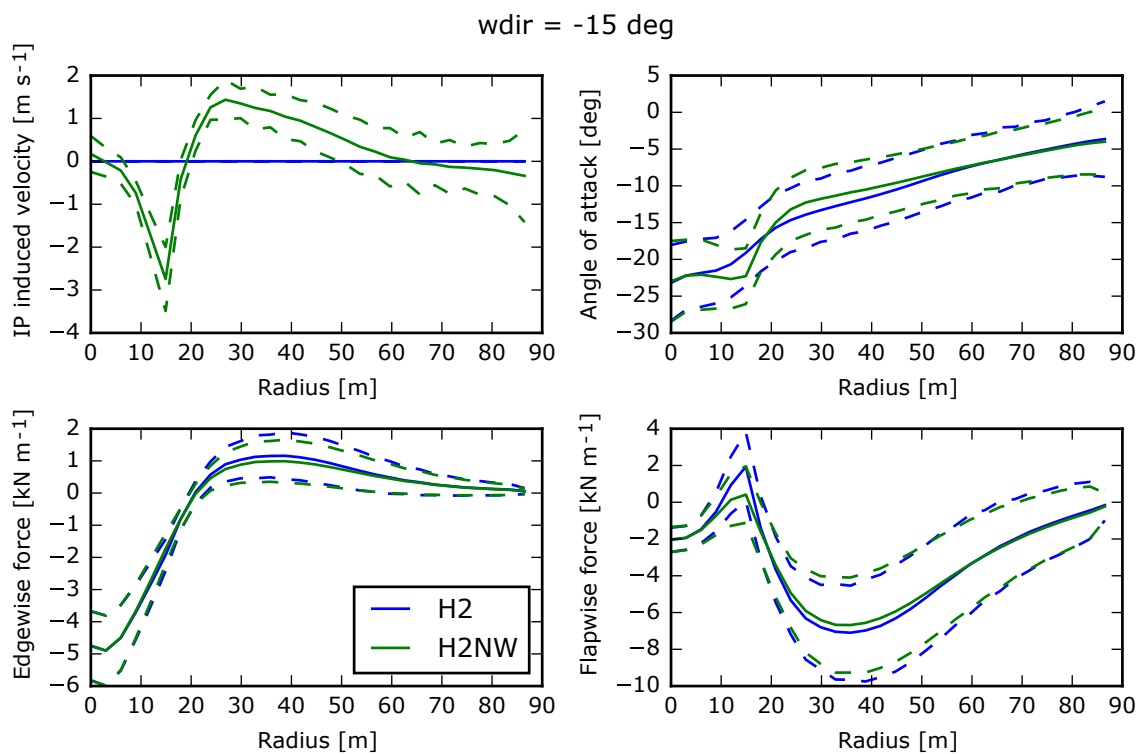

Figure 10. Distribution of induction, AOA, and loads for the upward-pointing blade. The mean values are shown as solid lines, and the dashed lines indicate the standard deviations; IP indicates in-plane.
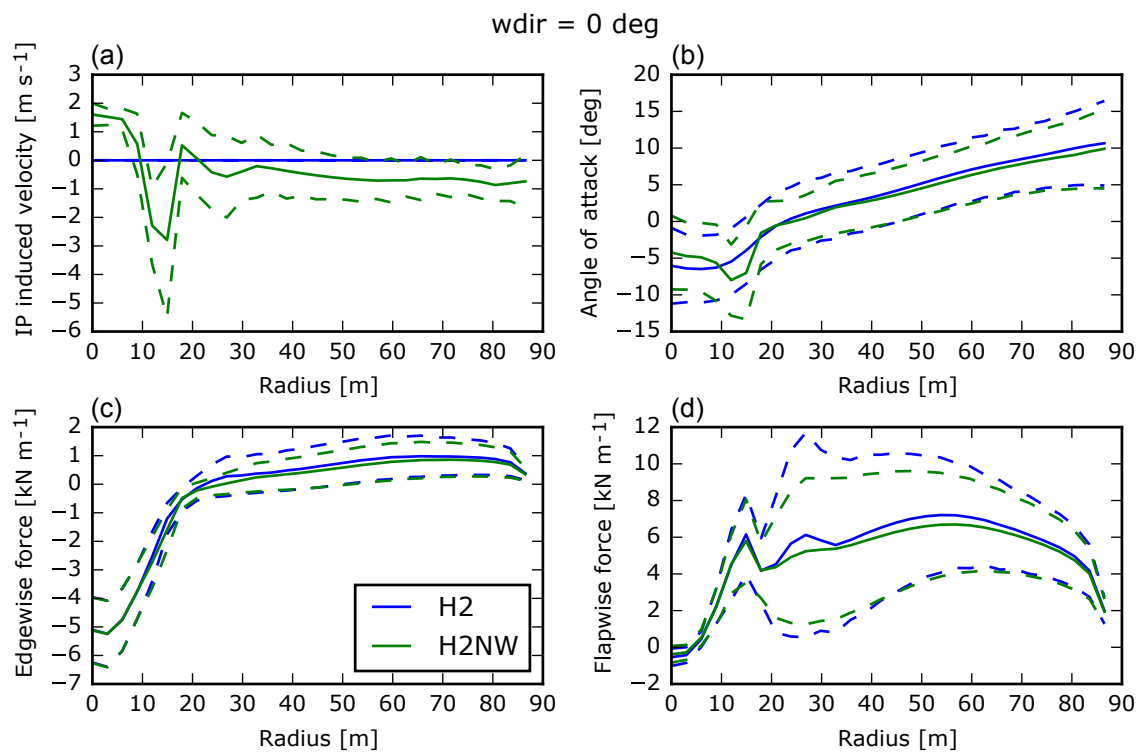

Figure 11. Distribution of induction, AOA, and loads for the upward-pointing blade. The mean values are shown as solid lines, and the dashed lines indicate the standard deviations.

almost the whole blade. The root vortex is clearly visible in the plot of the induced velocity in Fig. 11a.

The flow is stalled along the whole blade at $15^{\circ}$ wind direction. Therefore, the quite large induced velocities if the near-wake model is active have only a minor effect on the aerodynamic forces, even though using the near-wake model changes the mean local AOA by up to $5^{\circ}$; see Fig. 12 . The forces at the blade root, with around $25 \mathrm{~m}$ radius, are clearly smaller in the HAWC2 NW than in the HAWC2 computations, but because of the small lift gradient around stall, this reduction does not affect the standard deviations of the aerodynamic forces as much.

\subsubsection{Idling rotor}

Computations with 36 different turbulence seeds per wind direction and for each of the two aerodynamic models have been performed to investigate the influence of the aerodynamic model on the extreme flapwise and edgewise blade 


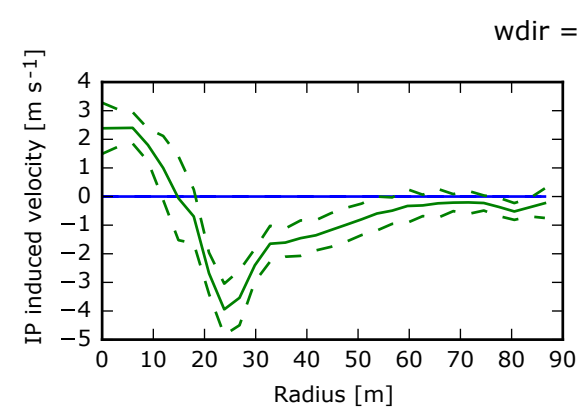

wdir $=15 \mathrm{deg}$
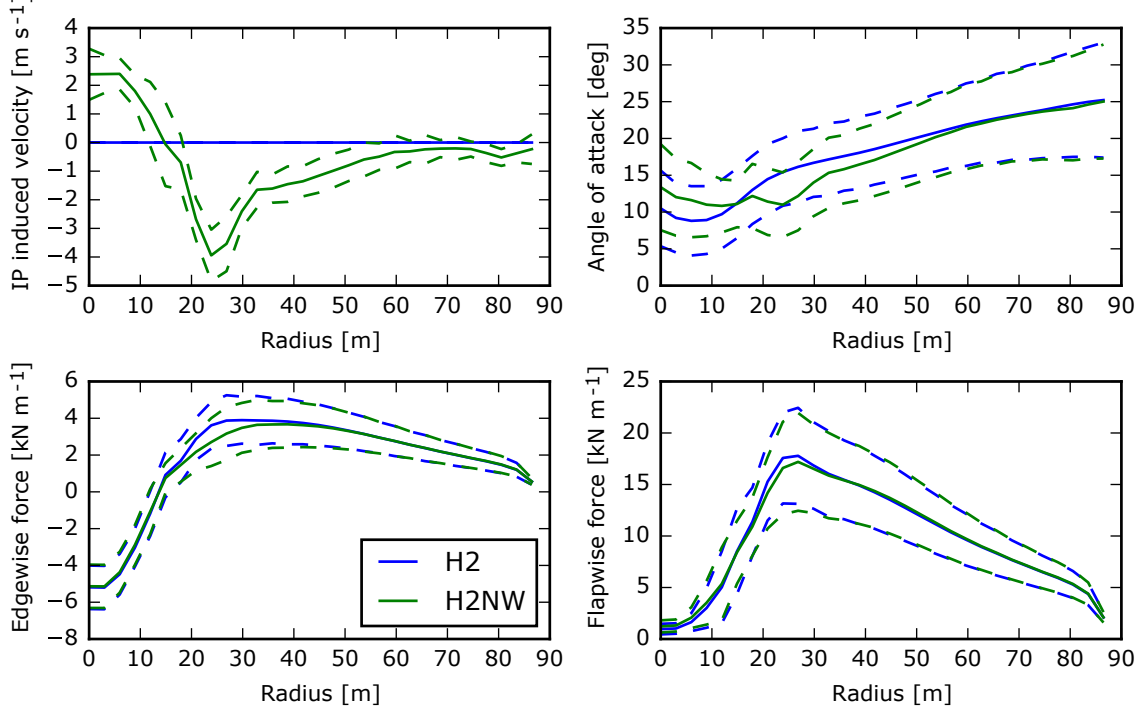

Figure 12. Distribution of induction, AOA, and loads for the upward-pointing blade. The mean values are shown as solid lines, and the dashed lines indicate the standard deviations.
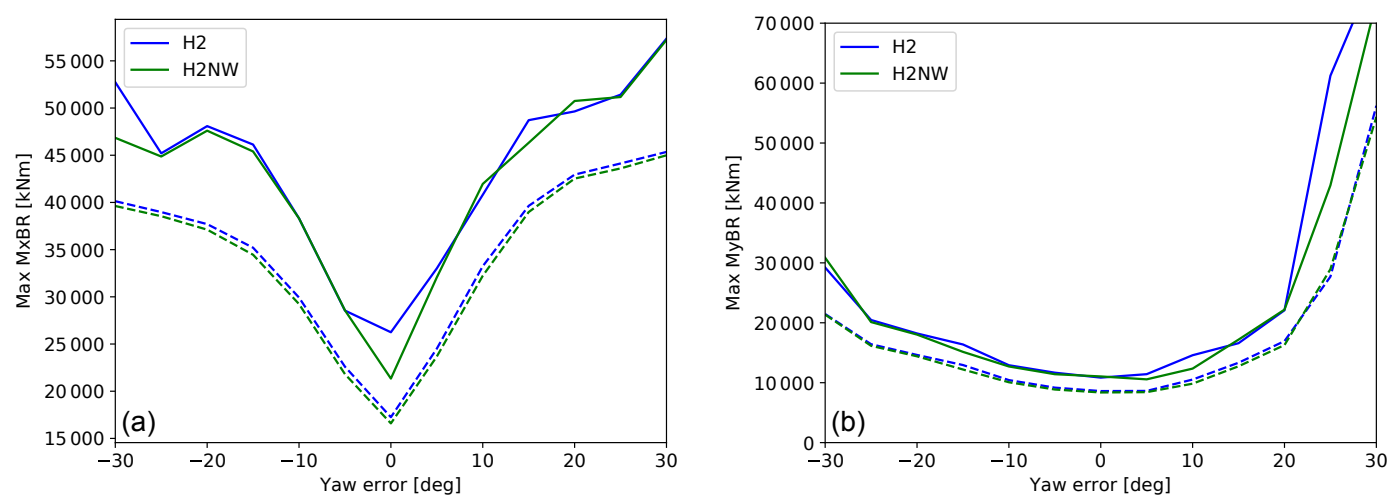

Figure 13. Maximum flapwise (a) edgewise and (b) blade root bending moment for wind directions between -30 and $30^{\circ}$ yaw error. Dashed lines show the mean of the maximum values for the 36 different seeds, the solid lines the maximum of the maxima.

root bending moment. The result of these simulations is shown in Fig. 13.

The dashed lines represent the mean value of the maximum absolute flapwise and edgewise blade root bending moment in the 36 simulations. The absolute maximum of the maxima encountered in the simulations is shown as solid lines. It can be seen that including the near-wake model in the simulations reduces the mean maximum value by roughly 0.5 to $1.5 \%$, depending on the wind direction. An exception for this reduction is the edgewise moment at $25^{\circ}$ yaw error, where vibrations are present and distort the analysis. The influence of the aerodynamic model on the absolute maximum encountered in the 36 seeds (see the solid lines in Fig. 13) is less clear. An explanation for this might be that in the idling cases with yaw error, the blades see different AOA distributions as a function of azimuth position. A very high flapwise blade root bending moment will occur if a high wind speed hits a large part of the blade at an angle of attack corresponding to the maximum lift coefficient. Because the lift gradient at the maximum lift coefficient is small, the influence of the near-wake model in this extreme case is small as well; therefore, the highest flapwise blade root bending moments at yaw error are very similar in HAWC2 and HAWC2NW. This extreme case of high wind speed at maximum lift coefficient does not occur with each turbulence seed though. If the extreme loading does not occur at the maximum lift coefficient in a 600 s HAWC2 simulation, then the trailed vorticity leads to a reduced maximum loading. Therefore, the average extreme loading of the simulations with 36 turbulence seeds is decreased when the near-wake model is active. At 25 and $30^{\circ}$ yaw error, the maximum edgewise blade root moments indicate edgewise vibrations. The near-wake model seems to 

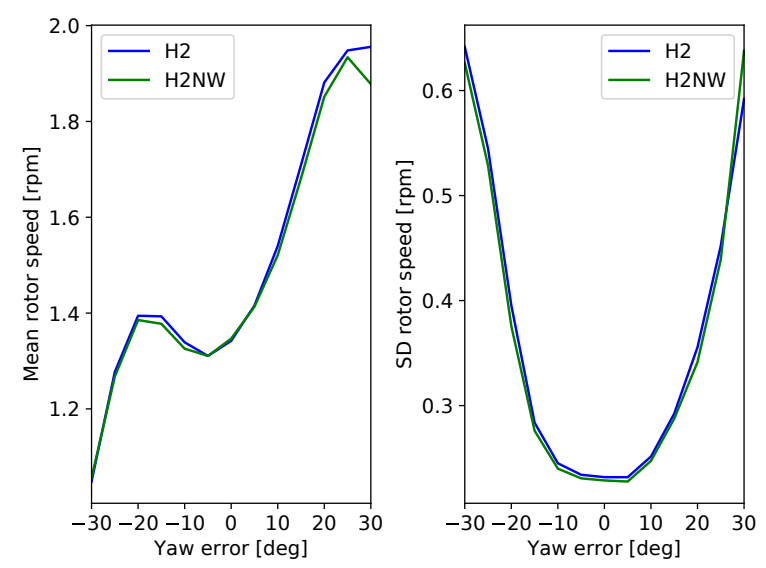

Figure 14. Mean and standard deviation of the idling rotor speed. The near-wake model slightly reduces mean idling rotor speed as well as its standard deviation, which is consistent with the generally lower flapwise loading predicted by HAWC2 NW.

reduce the absolute maximum of edgewise blade root bending moment encountered in the 36 seeds when vibrations are present, but the effect on the mean maximum values is smaller.

The mean values of the mean and standard deviation of the idling speed for the 36 seeds are shown in Fig. 14. If the near-wake model is active, HAWC 2 predicts both slightly lower idling speeds and lower idling speed variations. This is consistent with the generally slightly lower loading observed in Figs. 10 to 12.

The load comparison in idling conditions shows that adding the near-wake model might appear to reduce the extreme loading if a small number of turbulence seeds is used. A high number of turbulence seeds, on the other hand, is expected to lead to the same extreme loading independent of trailed vorticity model. Another conclusion is that the maximum extreme loading is much higher than the average extremes of the $10 \mathrm{~min}$ time series with different turbulence seeds. A large number of seeds might be necessary to achieve realistic extreme values in an aeroelastic load analysis in standstill conditions.

\section{Conclusions}

The near-wake model has been extended to compute the induction due to trailed vorticity in standstill and idling conditions. Due to the twist distribution of a wind turbine blade and the larger effect of turbulence in standstill when compared to operational conditions, strong vortices can be trailed from any position along the span of the blade. In idling conditions yaw errors, tilt angle, and wind inclinations directly translate into AOA variations on the slowly rotating blades. Comparison with the analytical solution of a constant downwash for an elliptical wing shows good agreement with results from the extended near-wake model, with the original model wrongly predicting large radial variations in the downwash.

Comparison with measurements from the NREL/NASA Ames Phase VI experiment in attached flow conditions shows an unexplained offset between the steady-state normal and tangential force coefficients measured and predicted by HAWC 2 NW. However, the HAWC 2 NW code predicts the effect of the trailed vorticity on the radial load gradients in steady state.

A comparison of the dynamic variation in the force coefficients for a sinusoidally pitching blade in attached flow shows that HAWC2 NW can predict dynamic loops that agree much better with the measurements than those predicted by HAWC 2 on the major part of the blade. The agreement is improved both in terms of $c_{n}$-AOA curve gradients and openings of the loops.

In a steady-state comparison at high mean AOA, where the flow is separated at most of the blade, the near-wake model can predict the root vortex at the inner part of the blade in attached flow. At the rest of the blade, no clear improvement due to the added trailed vorticity modeling is visible. At the tip, which is in deep stall, the predicted normal force coefficient agrees less well with the measurements.

The unsteady comparison at high AOA shows a clear improvement at the inner part of the blade, which is in attached flow. Also on the outer part the openings of the $c_{n}$ loops are predicted better by HAWC2 NW than HAWC2, mainly because the flow in the HAWC2 simulations is close to fully separated, where the dynamic stall model cannot predict the dynamic behavior. The HAWC2 NW simulations predict lower AOAs close to the blade tip, and thus the dynamic stall loops stay open. Even though the trailed vorticity modeling leads to improved predictions in this case, the basic weakness of the Beddoes-Leishman-type dynamic stall model in deep stall should be addressed in future research. Further, the outboard $c_{t}$ loops in stall are found to be difficult to model. However, the Unsteady Aerodynamics Experiment (UAE) Phase VI $c_{t}$ measurements, and $c_{t}$ measurements in general, are highly sensitive to pressure tap distribution, which can lead to increased uncertainties in this measurement.

As expected, the aeroelastic computations in standstill with turbulent inflow and a fixed rotor show that the nearwake model reduces the mean blade loading mainly at radial positions in attached flow compared to the standard standstill aerodynamic model without induction. The standard deviations of the force variations are reduced accordingly. Because the relative velocity in standstill is similar at all radial positions of the blade and the chord gets smaller towards the tip, no large tip loss effects have been observed and the main induction is clearly due to the root vortex.

Also computations with idling rotor in a yaw error range of -30 to $+30^{\circ}$ have been performed with 36 turbulence seeds per wind direction. The absolute maximum of flapwise blade root bending moment shows only a very small influence of 
the aerodynamic model. The mean maximum encountered with the different turbulence seeds shows a small but clear reduction due to the trailed vorticity modeling.

\subsection{Future work}

For a different turbine and more flexible blade design, standstill vibrations in attached flow can be possible. The impact of the trailed vorticity modeling on these vibrations could be addressed in future research.

The damping of vibrations in parked or idling conditions can also be highly dependent on the dynamic stall model parameters. The attached flow parameters used in HAWC2 are based on the analytical solution for the dynamic lift and drag of a flat plate, and the airfoil thickness could be taken into account here. Also the time constants for the flow separation are currently assumed to be independent of the airfoil, as well as identical in positive and negative stall. This assumption is certainly wrong for cambered airfoils, and a better approach could be identified in the future.

Leading-edge separation, which is not part of the current dynamic stall model implementation, could become very important at extreme yaw errors, where the AOA is around $180^{\circ}$. Then, the trailing edge of the airfoil acts as a sharp leading edge and leading-edge separation is much more likely than in the cases investigated in the present article.

Data availability. The measurement data are available by request from NREL using the relevant case numbers found in the article. The aerodynamic computations have been executed with HAWC2. Commercial and research licenses for HAWC2 can be purchased from DTU.

Competing interests. The authors declare that they have no conflict of interest.

Special issue statement. This article is part of the special issue "The Science of Making Torque from Wind (TORQUE) 2016". It is a result of the The Science of Making Torque from Wind (TORQUE 2016) conference, Munich, Germany, 5-7 October 2016.

Acknowledgements. The work has been conducted within the project "Research and development of optimal wind turbine rotors under offshore wind conditions in China (OffWindChina)", funded by "Det Strategiske Forskningsråd ved Programkomiteen for Bæredygtig Energi og Miljø”, contract 12-130590.

Edited by: Gerard J. W. van Bussel

Reviewed by: Xabier Munduate and Vasilis A. Riziotis

\section{References}

Bak, C., Bitsche, R., Yde, A., Kim, T., Hansen, M., Zahle, F., Gaunaa, M., Blasques, J., Døssing, M., Wedel Heinen, J., and Behrens, T.: Light Rotor: The 10-MW reference wind turbine, European Wind Energy Association (EWEA) Conference and Exhibition, 16-19 April 2012, Copenhagen, Denmark, 2012.

Hand, M., Simms, D., Fingersh, L., Jager, D., Cotrell, J., Schreck, S., and Larwood, S.: Unsteady aerodynamics experiment phase VI: wind tunnel test configurations and available data campaigns, NREL/TP-500-29955, National Renewable Energy Laboratory Golden, Colorado, USA, https://doi.org/10.2172/15000240, 2001.

Hansen, M., Thomsen, K., Natarajan, A., and Barlas, A.: Design Load Basis for onshore turbines - Revision 00, E-0074, DTU Wind Energy, Denmark, 2015.

Hansen, M. H., Gaunaa, M., and Madsen, H. A.: A BeddoesLeishman type dynamic stall model in state-space and indicial formulations, Ris $\emptyset-R-1354$, Roskilde, Denmark, 2004.

Johansen, J., Sørensen, N. N., Michelsen, J. A., and Schreck, S.: Detached-eddy simulation of flow around the NREL Phase VI blade, Wind Energy, 5, 185-197, https://doi.org/10.1002/we.63, 2002.

Larsen, T. J. and Hansen, A. M.: How 2 HAWC2, the user's manual, Denmark, Forskningscenter Risoe, Risoe-R-1597, available at: http://www.hawc2.dk/download/hawc2-manual (last access: 14 November 2017), 2015.

Madsen, H. A. and Rasmussen, F.: A near wake model for trailing vorticity compared with the blade element momentum theory, Wind Energy, 7, 325-341, https://doi.org/10.1002/we.131, 2004.

Pirrung, G. R., Hansen, M. H., and Madsen, H. A.: Improvement of a near wake model for trailing vorticity, Journal of Physics: Conference Series, 555, 012083, https://doi.org/10.1088/17426596/555/1/012083, 2014.

Pirrung, G. R., Madsen, H. A., Kim, T., and Heinz, J.: A coupled near and far wake model for wind turbine aerodynamics, Wind Energy, 19, 2053-2069, https://doi.org/10.1002/we.1969, 2016.

Pirrung, G., Riziotis, V., Madsen, H., Hansen, M., and Kim, T.: Comparison of a coupled near- and far-wake model with a free-wake vortex code, Wind Energ. Sci., 2, 15-33, https://doi.org/10.5194/wes-2-15-2017, 2017.

Sørensen, N. N. and Schreck, S.: Computation of the National Renewable Energy Laboratory Phase-VI rotor in pitch motion during standstill, Wind Energy, 15, 425-442, https://doi.org/10.1002/we.480, 2012.

Wang, K., Riziotis, V. A., and Voutsinas, S. G.: Aeroelastic Stability of Idling Wind Turbines, Journal of Physics: Conference Series, 753, 042008, https://doi.org/10.1088/1742-6596/753/4/042008, 2016.

Wang, T. and Coton, F. N.: A modified near wake dynamic model for rotor analysis, Aeronaut. J., 103, 143-146, https://doi.org/10.1017/S0001924000064952, 1999. 